ESTRATÉGIA 


\title{
EFEITO MEDIADOR DO DESEMPENHO OPERACIONAL NO ECO-DESIGN/LOGISTICA REVERSA
}

\author{
MEDIATION EFFECT OF OPERATIONAL PERFORMANCE \\ IN ECO-DESIGN/REVERSE LOGISTIC
}

Roberto Giro Moori

Universidade Presbiteriana Mackenzie

Fabio Ytoshi Shibao

Universidade Nove de Julho

\author{
Data de submissão: 25 jul. 2017. Data de aprovação: \\ 15 dez. 2017. Sistema de avaliação: Double blind review. \\ Universidade FUMEC / FACE. Prof. Dr. Henrique Cordeiro \\ Martins. Prof. Dr. Cid Gonçalves Filho.
}

\section{RESUMO}

Objetivo deste estudo foi o de estimar um modelo teórico empírico que contempla o desempenho operacional como fator mediador na relação entre eco-design/logística reversa e desempenho ambiental em empresas do ramo químico. Para tanto, realizou-se uma pesquisa constituída de duas etapas. A primeira, de natureza exploratória do tipo qualitativo, utilizou-se de uma amostra de 10 empresas cujos dados foram coletados por meio de entrevistas em profundidade. A segunda, de natureza descritiva do tipo quantitativo, precedida de sete pré-testes, utilizou-se de uma amostra de 316 empresas cuja coleta de dados se deu por meio de questionários semiestruturados. Os dados coletados, tratados pela modelagem em equações estruturais, revelaram que o desempenho operacional medeia a relação entre eco-design/ logística reversa e desempenho ambiental. Diante deste resultado pode-se concluir que o desempenho operacional teve um importante papel para atender o desempenho ambiental.

\section{PALAVRAS-CHAVE}

Eco-design. Logística Reversa. Desempenho Operacional. Desempenho Ambiental. Indústria Química. 


\section{ABSTRACT}

The objective of this study was to estimate an empirical theoretical model that contemplates the operational performance as a mediating factor in the relation between eco-design/reverse logistic and environmental performance in chemical companies. For this, a research was carried out in two stages. The first, a study of exploratory nature of the qualitative type, that was used a sample of 10 companies whose data were collected through in-depth interviews. The second, a study of descriptive nature of the quantitative type, preceded by seven pre-tests, was used a sample of 316 companies whose data collection was done through semi-structured questionnaires. The collected data, treated by modeling in structural equations, revealed that the operational performance mediates the relationship between eco-design/reverse logistic and environmental performance. In view of this result, it can be concluded that the operational performance played an important role in meeting environmental performance.

\section{KEYWORDS}

Eco-design. Reverse Logistic. Operational Performance. Environmental Performance. Chemical Industry.

\section{INTRODUÇÃO}

Eco-design é um termo que tem ganhado destaque no mundo dos negócios, sociedade e governo, especialmente, a partir da Conferência das Nações Unidas sobre - Meio Ambiente e o Desenvolvimento, ocorrida na cidade do Rio de Janeiro em 1992, conhecida como ECO-92, em que mais de cem países, incluindo o Brasil, assinaram a Agenda 21, que propõe práticas e técnicas de desenvolvimento sustentável para nações, estados e cidades.

Esse termo, com forte relação com a sustentabilidade ambiental, tem a finalidade de reduzir impactos ambientais de produção durante o seu ciclo de vida (ZHU; SARKIS, 2007). Segundo Corrêa e Corrêa (20I2), o impacto ambiental começa a ser definido a partir do momento em que uma equipe de desenvolvimento de produto e de processos de fabricação coloca pela pri- meira vez sua lapiseira sobre a prancheta, ou o ícone do mouse na tela do computador, ainda em branco.

Embora, o eco-design seja uma necessidade inquestionável para a sustentabilidade ambiental, nem sempre é possível projetar um produto totalmente ecológico. Uma porcentagem muito grande de produtos, ainda não são resolvidos pelo eco-design como as pilhas, as baterias e as embalagens plásticas. Nesse sentido, segundo a Política Nacional de Resíduos Sólidos (PNRS, 20I2), a gestão ambiental, deve seguir a seguinte ordem de prioridade: não geração de resíduos, redução, reutilização, reciclagem, tratamento dos resíduos sólidos e disposição final ambientalmente adequada dos rejeitos.

Por conta disso, a logística reversa assume importância fundamental no eco-design, definida pela PNRS (20I2) como "um 
instrumento de desenvolvimento econômico e social caracterizado por um conjunto de ações, procedimentos e meios destinados a viabilizar a coleta e a restituição dos resíduos sólidos ao setor empresarial, para reaproveitamento, em seu ciclo ou em outros ciclos produtivos, ou outra destinação final ambientalmente adequada".

Ao lado da eco-design e da logística reversa, outro elemento importante é a prática da produção mais limpa, cuja finalidade é reduzir a carga ecológica nos processos produtivos mediante o uso apropriado dos materiais e tecnologias de processo para melhorar a eficiência energética (UNEP, 200I) e obter, simultaneamente, desempenhos operacionais e ambientais. Os tomadores de decisão, em todos os níveis, para prevenir a poluição em processo, produtos e serviços, estão percebendo que é mais vantajoso desenvolver atividades, de forma integrada, em vez de controlá-los após a ocorrência do fato. Nesse sentido, ferramentas administrativas como análise do ciclo de vida, tecnologias de fabricação que economizam energia e sistemas de gestão ambiental, devem ser adotadas para não gerar desperdícios e conservar os recursos naturais (LARDEREL, 200I).

De fato, a melhor etapa, embora não seja a única, para se ter um produto ou processo produtivo que causa baixo, ou nenhum, impacto ambiental é o eco-design. Nessa etapa, a empresa pode atuar nos potenciais problemas ambientais que ocorrem no processo produtivo (CORRÊA; CORRÊA, 20I2) e, na impossibilidade de evita-los, desenvolver o devido sistema de logística reversa.

Assim, baseado nessa premissa foi formulado a seguinte questão de pesquisa: o desempenho operacional medeia a rela- ção entre eco-design/logística reversa e o desempenho ambiental? $\bigcirc$ objetivo foi o de estimar um modelo teórico-empírico que incorpora o desempenho operacional como um fator mediador da relação entre eco-design/logística reversa e o desempenho ambiental.

O estudo está estruturado da seguinte maneira. Após a introdução, expôs-se o referencial teórico e hipóteses. No item 3 são descritos os procedimentos metodológicos seguidos das análises dos dados e resultados. Por fim são apresentados as conclusões e sugestões para prosseguimento do estudo.

\section{REFERENCIALTEÓRICO E HIPÓTESES}

Compreender como se eleva a ecodesign/logística reversa a uma posição de destaque, é de grande relevância para as empresas, dado que, ela contribui para a sustentabilidade ambiental. Há evidências empíricas de que a eco-design/logística reversa é um antecedente do desempenho ambiental (ZHU; SARKIS, 2004; TIBBEN -LEMBKE, 2002). Todavia, os resultados sobre o tipo de relação direta existente entre essas duas variáveis [eco-design/logística reversa $\rightarrow$ desempenho ambiental] têm sido inconclusivos (ZHU; SARKIS, 2004). Nesse sentido, esse estudo investiga a existência de uma variável, representada pelo desempenho operacional, atuando como mediadora nessa relação. Entender o papel mediador do desempenho operacional nessa relação, justifica-se ao fato, de que 'toda relação assimétrica entre duas variáveis' é uma abstração feita a partir de uma interminável cadeia causal e quanto maior compreensão se obtém acerca dessas variáveis, melhor será a gestão acerca dessa 
relação.Assim exposto, construiu-se o modelo teórico empírico conforme é mostrado na Figura I.

O suporte teórico e hipóteses que sustentam o modelo são descritos a seguir.

\section{Sem Mediação - caminho (a)}

\section{A Influência do Eco-Design/Logística Reversa no Desempenho Ambiental}

Eco-design é um conceito que integra aspectos multifacetados de projetos, processos produtivos e aspectos ambientais com o objetivo de desenvolver produtos ambientalmente sustentáveis que satisfaz as necessidades e desejos humanos (ISO I 4006, 20 I I; KIURSKI, et al., 20I7).

Srivastava (2007) destaca a eco-design segundo duas dimensões: projeto ambientalmente consciente (Environmentally Conscious Design - ECD) e analise/avaliação do ciclo de vida do produto (LifeCycle Analysis/Assessment - LCA). Os objetivos, de ambas as dimensões, são os de reduzir o impacto ambiental. Enquanto na $E C D$, o projeto do produto está associado a recuperação de materiais, desmontagem, legislação, minimização de perdas, remanufatura, reciclagem e melhor escolha de materiais; a LCA está associada ao processo de produção para analisar e avaliar o meio ambiente, a fabricação, o transporte, a distribuição, uso, reciclagem e a disposição final do produto.

Essas duas dimensões leva a necessidade de perceber a importância da logística reversa. Não se trata da logística reversa tradicional que se dedica ao retorno de materiais de pós-venda ou de pós consumo (LEITE, 20I7), mas da logística reversa que se origina no projeto do produto. Nesse sentido, as empresas precisam perceber o custo oculto na logística reversa e focar nessa função (MOLLENKOPT; CLOSS, 2005, SRIVASTAVA, 2007) para conhecer o impacto financeiro e desenvolver estratégias de logística reversa.

Nesse sentido a eco-design/logística reversa como atividades integradas que ocorrem dentro de áreas funcionais de empresas individuais, que se prolongam para além das fronteiras organizacionais, por meio de conexões com parceiros (fornecedores e clientes), sociedade e governo, permite alargar a visão estreita da eco-design (SRIVASTAVA, 2007). Compreender essa visão integrada da eco-design, sob a ótica da logística reversa pode considerada fonte importante de inspiração da sustentabilidade (UNEP, 200I).

Em se tratando de competição global, o planejamento e o projeto do produto, cujos componentes ou matérias-primas utilizados causam menor impacto ambiental, são cruciais para o sucesso da empresa. Não obstante, integrar aspectos ambientais no processo de desenvolvimento de produto requer mudanças gerenciais, técnicas e atividades operacionais para conceber "um projeto de produto verde" (KRUWET; ZUSSMAN; SELIGER, (995).

A evolução da visão tradicional do ciclo de vida do produto para a abordagem do berço ao berço (from cradle to cradle), para ser competitiva, requer um processo logístico reverso, dado que, inovações ecológicas depende do gerenciamento eficaz dos fluxos reversos dos materiais (NINLAWAN et al., 2010).

Estabelecer metas para implementação de produtos Eco-design e logística reversa permite ao administrador, avaliar desempenhos em uso de matérias-primas menos agressivas ao meio ambiente, de tal modo a reduzir o consumo de material e energia 
no processo de fabricação; de transporte e distribuição; de embalagem; e de custos em logística reversa do produto (NINLAWAN et al., 20 I 0; ZHU; SARKIS, 2004; ZHU et al., 2010). Em geral, segundo a norma NBR-ISO:|4040-200I, o desempenho ambiental avalia aspectos da gestão ambiental.Tipicamente não aborda aspectos econômicos ou sociais de um produto. Assim, é de se esperar:

$\mathrm{H}_{1}$ : Eco-Design/Logística Reversa impacta diretamente no Desempenho Ambiental Com Mediação - Caminhos: (b) e (c)

\section{A Influência do Eco-Design/ Logística Reversa no Desempenho Operacional - Caminho (b)}

Eco-design/Logística reversa, na dimensão da LCA, envolve redução de resíduos, aumento da reciclagem, diminuição da emissão de gases do efeito estufa, eliminação do produto de volta ao meio ambiente no fim da vida, ou o seu retorno à fabricação, isto é, o gerenciamento do produto do 'berço ao berço' (from cradle to cradle). A diminuição dos resíduos na produção é uma das estratégias mais visíveis que estão sendo implementadas pelas empresas por meio dos processos de produção enxuta (BOWERSOX et al., 20l4) que também tem promovido o uso eficiente dos materiais (ROMM, 1994). Além disso, as empresas procuram melhorar os processos produtivos, implementar ferramentas de gestão para as práticas de produção mais limpa para reconciliar desenvolvimento econômico com proteção ambiental (TOEPFER, 200I).

Pela perspectiva da gestão da cadeia de suprimentos, a conservação eficaz e a administração dos recursos envolvem diversas funções interorganizacionais. Gerenciar melhor e minimizar a dependência dos recursos energéticos, da água e de outros recursos naturais,

a) Sem Mediação

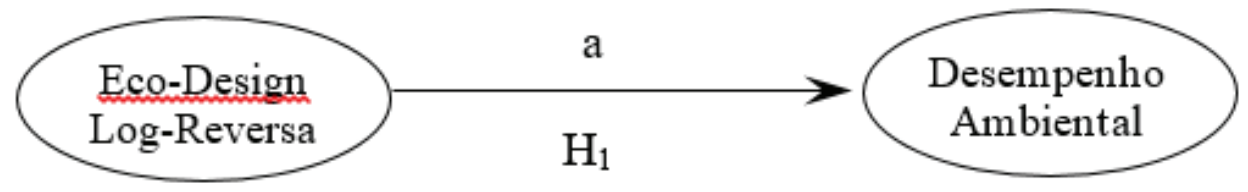

b) Com Mediação

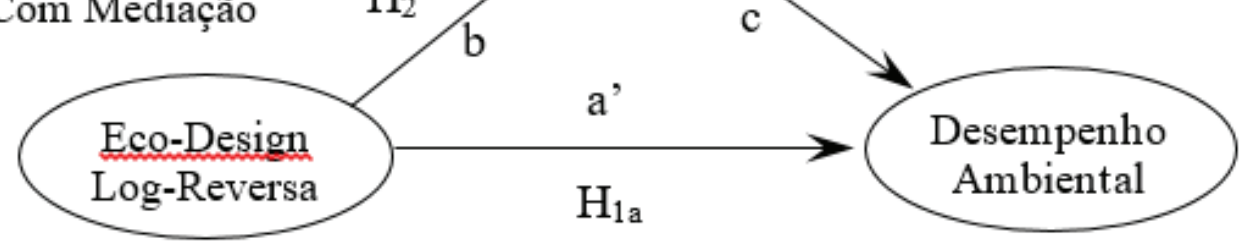

FIGURA 1 - Modelo teórico-empírico da pesquisa

Fonte: Os autores 
requer substituição de materiais, reconfiguração de arranjo físico no interior da fabricação, tecnologias, capacitação e treinamento de pessoas (BOWERSOX et al., 20I4).

Segundo Linton, Klassen e Jayaraman (2007), dada a convergência de cadeias de suprimentos e sustentabilidade, o foco da gestão ambiental e de operações, são movidas para a otimização dos fatores ambientais locais para a cadeia de suprimentos durante a produção, o consumo, o atendimento ao cliente e a disposição dos descartes de produtos.

No contexto da gestão da cadeia de suprimentos, os transportes rodoviários, importantes elementos de conexões entre os membros, são reconhecidos como uma das barreiras mais significantes para a sustentabilidade, particularmente nas áreas urbanas, pelo consumo expressivo de combustíveis fósseis, emissão de poluentes, ruídos e congestionamentos. Todavia, movimentação de produtos, em áreas urbanas, é fundamental para a geração de riqueza. Nesse sentido, Bowersox et al. (20|4), argumentam que o equilíbrio (trade-offs) entre o impacto ambiental negativo e a geração de riqueza podem ser gerenciados pelo uso de período definido de movimentação, carga e descarga na área urbana e por projetos para a logística reversa.

Por conta disso, os trade-offs das medidas de desempenho operacionais tradicionais são criticadas porque focam em resultados de curto prazo, apresentam pouco incentivo às inovações e encorajam apenas meIhorias locais (NEELY, 1998). Por exemplo, o incentivo dado aos gestores para manter equipamentos em produção para elevar o desempenho da utilização de recursos, sem considerar o aumento do estoque, pode elevar os custos. Todavia, existem argumentos, ao contrário, como a reação em cadeia de Deming (2000) que argumenta, a melhoria da qualidade reduz os custos uma vez que os desperdícios são reduzidos ou mesmo eliminados.

Nesse sentido cabe à administração estabelecer mecanismos ou práticas produtivas, como a produção mais limpa, para prevenir a poluição, diminuir riscos e melhorar a ecoeficiência (SANT'ANA; CAMPOS; PEREIRA, 20I7), ao invés de ações de fim de tubo (end-of-pipe) que não oferece salto qualitativo tecnológico para reduzir a emissão de poluentes na fonte. Como exemplo, têm-se os estudos ambientais em empresas japonesas realizados por Zhu et al. (20l0). Esses autores, citam duas implementações de design ecológico de produtos com diferentes focos na regulação industrial japonesa. A primeira, para eliminar ou reduzir o uso de materiais perigosos no processo de fabricação com impacto no desempenho ambiental; e a segunda, relacionados a investimentos para recuperar sucata de materiais usados com impacto no desempenho operacional. Assim, é de se esperar:

$\mathrm{H}_{2}$ : Eco-Design/Logística Reversa impacta diretamente no Desempenho Operacional

\section{A Influência do Desempenho Operacional no Desempenho Ambiental - Caminho (c)}

Desempenho operacional refere-se ao cumprimento de metas operacionais, normalmente com ênfase no curto prazo (HALL, 2004). Um sistema para avaliar o desempenho exige uma perspectiva funcional (BOWERSOX, CLOSS e COOPER, 2006). Dentro dessa perspectiva funcional sobressaem dimensões como: custos, qualidade do produto e confiabilidade, velocidade e confiabilidade na entrega. A lógica fundamental é de que uma operação não 
pode ter um desempenho excelente em todas essas dimensões (CHASE, JACOBS e AQUILANO, 2006).

Com o despertar das questões ambientais, notadamente, o desempenho ambiental foi incorporado às organizações, que a norma NBR ISO:I400I-2004 o define como resultados mensuráveis, da gestão de uma organização, sobre seus aspectos ambientais. Acrescenta ainda, segundo a norma NBR ISO: I 400 I-2004, no contexto de sistemas de gestão ambiental, os resultados podem ser medidos com base na política ambiental, objetivos ambientais, metas ambientais da organização e outros requisitos de desempenho ambiental.

Diante do exposto, observa-se que, enquanto o desempenho operacional evidencia decisões de curto prazo, o desempenho ambiental exige tomadas de decisão de maior prazo. Ainda, conforme recomendações da norma NBR ISO:I400I-2004, que a organização avalie quais de suas operações estão associadas com seus aspectos ambientais significativos identificados e assegure que elas sejam conduzidas de modo a controlar ou reduzir os impactos ambientais adversos associados, para atender aos requisitos de sua política ambiental e atingir seus objetivos e metas. Colocando de outro modo, o desempenho operacional é muito mais pressionado do que o desempenho ambiental, o que poderia perturbar a previsibilidade das empresas em atender as metas ambientais.Assim, é de se esperar que:

$\mathrm{H}_{3}$ : Desempenho Operacional impacta diretamente no Desempenho Ambiental

\section{Mediação - ligação [Eco-design I Logística Reversa e Desempenho Ambiental] via [Desempenho Operacional]}

Apesar do eco-design/logística reversa ser considerado antecedente do desempenho ambiental, esta ligação pode não ser assim tão simples e direta, pois existem situações em que um aumento no eco-design/logística reversa pode não resultar necessariamente num nível de desempenho ambiental mais elevado. Trabalho como de Zhu e Sarkis (2004), em empresas chinesas em contexto de gestão da cadeia de suprimentos verde, evidenciaram que pressões institucionais, dado pelo mercado, regulação e competição, tiveram efeito moderador na relação entre eco-design/logística reversa e desempenhos ambiental e econômico (positivo e negativo).

Outros estudos, como os realizados por Vijayvargy e Agarwal (20I4), em empresas indianas, também em contexto de gestão da cadeia de suprimentos verde, evidenciaram que eco-design/logística reversa tiveram efeito direto sobre os desempenhos operacional e ambiental, mas não foi evidenciado efeito direto no desempenho financeiro.

Observa-se, contudo, que gestão ambiental é complexo e envolve implicações estratégicas. Como exemplo, Ren (200I) mostrou que a poluição ambiental era uma consequência de atividades econômicas, como estabelecimento de estratégias de investimentos em tecnologias para atuar nos processos produtivos e minimizar a geração de poluentes. Entretanto, custos ambientais em economia capitalista são tipicamente não totalmente internalizados. Em termos de ambiente, em algumas ocasiões, privatização de propriedades é um bom instrumento para a administração de recursos comuns como florestas e terras, no entanto, elementos ambientais como o ar, água em áreas de livre acesso são impossíveis de definir suas fronteiras, mesmo para com regulação ambiental. 
Em termos de fabricação, é necessário a prática de um pensamento holístico, ou uma perspectiva de integração entre gestão ambiental e operacional para construir uma estrutura de administração unificada que inclui aspectos ambientais e operacionais. Alerta, entretanto, UNEP (200I), a introdução de novas ferramentas de gestão como a produção mais limpa, e de tecnologias de baixo consumo de energia são contribuições importantes do chão de fábrica na integração do eco-design/logística reversa e o desempenho ambiental.Assim, é de se esperar:

$\mathrm{H}_{\mathrm{I}}$ : Desempenho operacional medeia a relação entre Eco-Design/Logística reversa e desempenho ambiental

\section{PROCEDIMENTOS METODOLÓGICOS Natureza e Tipo de Pesquisa, Instrumento de Coleta de Dados e Sujeito da Pesquisa}

Este estudo foi considerado de natureza descritivo do tipo quantitativo. Para a construção do instrumento de coleta, previamente, foi realizado um estudo exploratório do tipo qualitativo junto a 10 empresas do ramo químico junto a gestores da cadeia de suprimentos verde. Os resultados do estudo exploratório resultaram em um questionário composto de três blocos. Os dois primeiros blocos referiram-se ao perfil, dos respondentes e das empresas, que fizeram parte da amostra. $O$ terceiro bloco referiu-se aos construtos eco-design/logística reversa, desempenho operacional e desempenho ambiental, sendo que cada um deles, foram compostos de cinco medidas.

Após o estabelecimento da primeira versão do questionário, submeteu-o a sete pré-testes por meio de entrevistas pesso- ais, para identificar e eliminar problemas potenciais como entendimento do enunciado, sequência, formato e instruções de preenchimento. As respostas obtidas com os pré-testes foram analisadas a verificada a confiabilidade de incorporação, definiu-se a construção do instrumento de coleta.

Com a definição do questionário, selecionou-se uma amostra, por acessibilidade, extraída de uma lista de empresas químicas sediadas no Brasil. Os questionários foram enviados, via e-mail, aos gestores que desenvolviam atividades relacionadas a suprimentos, engenharia, meio ambiente, logística e produção.

\section{Tratamentos dos Dados, Delimitações do Estudo e Limitações do Método}

Os dados coletados foram examinados pela estatística descritiva como frequência de resultados, média, desvio padrão, formato da distribuição e coeficiente de variação. Os valores considerados outliers foram retirados. Após o exame dos dados coletados, utilizou-se da estatística multivariada, a técnica da análise fatorial, para depurar e validar as medidas e escalas dos construtos do modelo de mensuração teórico-empírico, por meio da aplicação de testes de unidimensionalidade, confiabilidade composta e validações de conteúdo, convergência e discriminante. Validada as medidas, utilizouse da modelagem em equações estruturais para estimar a significância estatística das relações lineares do modelo de mensuração teórico-empírico. Em sequência, buscou-se verificar o ajuste do modelo teórico aos dados coletados empiricamente. Para a adequação dos dados ao modelo, foi utilizado o coeficiente de determinação o $R^{2}$, e para o ajuste global, o índice GoF (Godnes- 
s-of-Fit), dado por [ $G o F=)$ ], a despeito de Henseler e Sarsted (20I2) sugerirem não utilizar o índice GoF, por não ter o poder de distinguir modelos válidos e modelos não válidos. Ainda, foram utilizados a relevância ou validade preditiva, o indicador de Stone-Geisser $\left(\mathrm{Q}^{2}\right)$ e tamanho do efeito, o indicador de Cohen $\left(\mathrm{f}^{2}\right)$.

Por fim, para avaliar o papel mediador do desempenho operacional na relação entre eco-design/logística reversa e desempenho ambiental, utilizou-se da abordagem de lacobucci et al. (2007), por considera-la mais apropriada para tratar dados multivariados do que a abordagem de Baron e Kenny (I986). Como contraprova do resultado, foi utilizado o teste $z$ de Sobel, dado por: $z$ = $\rightarrow$ [Equação I], para avaliar a magnitude dos coeficientes dos caminhos (a e a') mostrados na Figura I, isto é antes e após o efeito da mediação. Ainda, utilizou-se da abordagem de lacobucci et al. (2007) para verificar a tipificação da mediação, se foi completa, parcial ou de supressão (sem mediação).

Como suporte ao tratamento dos dados, foi utilizado o software PLS-PM (Partial Least Square - Path Method) versão 2.0.

Delimitações do Estudo. Este estudo apresentou duas delimitações básicas. A primeira, se deu quanto ao escopo. A maioria das empresas da amostra estavam sediadas na região sudeste do Brasil, cujas principais empresas eram dotadas de competências tecnológicas robustas. A segunda delimitação, referiu-se à concepção da pesquisa, que foi transversal, ou seja, envolveu uma amostra de empresas cuja coleta de dados se deu somente uma vez.

\section{Limitações do Método da Pesqui-} sa. $O$ método escolhido para a pesquisa também apresentou duas limitações. A primeira, referiu-se ao porte das empresas químicas foram variados com diferentes tecnologias de processo. Nesse sentido, as empresas de maior porte levam vantagem, isto é, as respostas tendem para o lado concordante da escala, em relação a de menor porte, pelo uso intensivo de conhecimento, ciência, tecnologia, engenharia, mão de obra qualificada, o que pode ter introduzido algum viés nas respostas dos entrevistados.

A segunda, referiu-se à coleta de dados, na proporção de I:I isto é, um respondente uma empresa. Como as empresas eram constituídas de portes e tecnologias variadas, um único respondente para empresa também pode enviesar a resposta. Todavia, dado que foram obtidos 316 respondentes, acreditou-se que esse problema foi minimizado.

\section{ANÁLISES DOS DADOS E RESULTADOS \\ Perfil Demográfico da Amostra}

Os dados foram coletados no segundo semestre de 2012. Foram obtidos 316 questionários aptos para serem analisados que apresentaram os perfis demográficos descritos a seguir.

a) Com relação os respondentes. Os respondentes que fizeram parte da amostra, $27,2 \%$ estavam na função havia menos de 2 anos, $28,5 \%$ entre 2 a 5 anos, e $44,3 \%$ estavam na função havia cinco anos ou mais. Quanto ao tempo na empresa, $15,5 \%$ estavam na empresa havia menos de 2 anos, $24,4 \%$ entre 2 e 5 anos, e 60, I\% estavam na empresa havia a mais de cinco anos. Para o grau de escolaridade, os respondentes da amostra, 15,8\% tinham curso superior incompleto, e $84,2 \%$ tinham curso 
superior completo. Quanto ao exercício do cargo, $28,2 \%$ dos respondentes eram diretores e gerentes, e 7l,8\% nos demais cargos como engenheiros, superiores e coordenadores.

Em resumo, os respondentes foram compostos de pessoas com curso superior, com mais de cinco anos de tempo de empresa e exercício de função. Nesse sentido, os perfis dos respondentes denotaram que os dados coletados apresentavam boa confiabilidade para a análise.

b) Com relação às empresas. As empresas que fizeram parte da amostra, $45,6 \%$ estavam sediadas na região metropolitana da cidade de São Paulo, $20,9 \%$ no interior do estado de São Paulo e as demais, 33,5\% estavam localizadas nos estados do Rio Grande do Sul (7,6\%), Rio de Janeiro $(7,0 \%)$, Minas Gerais $(5,7 \%)$ e, em menor porcentagem, nos demais estados como Paraná, Santa Catarina e Bahia. As empresas, do total, 40,8\% tinham um faturamento de até $r \$ 10,5 \mathrm{mi}$ Ihões anuais, $16 \%$ entre $r \$ 10,5$ e 60 milhões e $43,2 \%$ tinham faturamento acima de $r \$ 60$ milhões anuais. Quanto ao número de empregados, $47,2 \%$ tinham até 99 empregados, $34,8 \%$ tinham entre 100 e 499 empregados e 10,8\% tinham acima de 500 empregados. Quanto ao ramo de atividades, $44,6 \%$ eram fabricantes de produto químico industrial e os demais eram fabricantes de produtos farmacêuticos, tintas, esmaltes, vernizes, defensivos agrícolas, adubos e fertilizantes. Quanto às certificações de qualidade, $72,2 \%$ tinham certificação ISO 9000 e $28,2 \%$ tinham a certificação ISOI 4000.
Em resumo, a amostra foi constituída por empresas localizadas principalmente na região sudeste do Brasil, com faturamento superior a $r \$ 60$ milhões anuais e acima de 100 empregados.

\section{Validação das Medidas e Escalas dos Constructos}

Para validar as medidas e escalas dos dados coletados junto a 316 respondentes, inicialmente, os dados foram submetidos a um exame descritivo para identificar as observações atípicas ou respostas extremas que poderiam influenciar o resultado da análise multivariada. Realizado o exame dos dados utilizou-se do software SmartPLS 2.0 para aplicar a técnica da análise fatorial. Após várias rodadas e interações, obtevese uma estrutura subjacente composta de 15 assertivas com cargas fatoriais superiores a 0,70 , cujo valor recomendado é de 0,7 , distribuídas em três construtos com 5 medidas cada, denominados: Eco-design/ Logística reversa, Desempenho Operacional e Desempenho Ambiental. As médias, desvios padrão, formato da distribuição (achatamento e alongamento) e cargas fatoriais de cada uma das assertivas, depuradas em seus respectivos constructos, são mostradas na Tabela IA no Apêndice I.

As estruturas dos constructos obtidas pela técnica da análise fatorial foram validadas quanto: a) a unidimensionalidade, dado pelo coeficiente $\alpha$-Cronbach, e a Confiabilidade Composta (CC), ambas as medidas, apresentaram valores acima do mínimo recomendados de 0,70 ; b) a validade convergente, avaliada pela carga fatorial e variância média extraída (VME) também obtiveram valores acima do mínimo recomendados de 0,7 e 0,5 , respectivamente. Salienta-se, todavia, que para o 
constructo Eco-design/Logística Reversa foi obtido VME de valor igual a 0,48, que é um valor muito próximo de 0,5, e; c) a validade discriminante, dada pelas variâncias compartilhadas e obtidas pela raiz quadrada da variância média extraída de cada construto, foram maiores que as correlações entre os constructos, conforme é mostrado na Tabela I (na diagonal da matriz em itálico), atendendo assim, a recomendação de Fornell e Larker (I98I).

Os dados da estatística descritiva como média e desvio padrão dos constructos, correlação bivariada, quantidades de assertivas originais e quantidades de assertivas após a depuração, medidas de $\alpha$-Cronbach, Variância Média Extraída (VME) e Confiabilidade Composta (CC) são mostrados na Tabela I.

Assim, validados as estruturas dos construtos com suas medidas e escalas prosseguiu-se na avaliação das relações estruturais do modelo de mensuração.

\section{Avaliação das Relações Estruturais do Modelo de Mensuração}

Os resultados, obtidos com o aplicativo SmartPLS 2.0, a técnica do PLS-PM, estão ilustrados na Figura 2.

Na Figura 2, são observados os coefi- cientes estruturais e, entre parênteses, os valores $\mathrm{t}$ dos relacionamentos entre os construtos [Eco-Design/Logística Reversa $\rightarrow$ Desempenho Operacional], [Eco-Design/Logística Reversa $\rightarrow$ Desempenho Ambiental] e [Desempenho Operacional $\rightarrow$ Desempenho Ambiental]. No modelo 'sem mediação', o construto Eco-Design/ Logística Reversa apresentou um efeito direto sobre o desempenho ambiental com coeficiente estrutural de 0,48 e estatística $t$ igual a 9,9l e suportou a hipótese $\mathrm{H}_{1}$.

No modelo ‘com mediação', o construto Eco-Design/Logística Reversa apresentou um efeito direto sobre o desempenho ambiental com coeficiente estrutural de 0,06 e estatística t igual a I,12 e não suportou a hipótese $\mathrm{H}_{\mathrm{la}}$. $\mathrm{O}$ efeito indireto foi igual a $0,42[0,58 * 0,72=0,42]$. $O$ efeito total foi igual a 0,48 $[0,06+0,58 * 0,72=0,48]$. A proporção da mediação do desempenho ambiental na variância do desempenho operacional explicada, via direta e indiretamente, pela Eco-design/Logística reversa foi de $87,4 \%[(0,58 * 0,72) /((0,58 * 0,72)+$ $0,06)=0,874]$. $O$ constructo Eco-Design/ Logística Reversa teve um efeito direto no Desempenho Operacional com coeficiente estrutural igual a 0,58 e estatística $t$ igual a 14,43 e suportou a hipótese $\mathrm{H}_{2}$, que por

TABELA 1 - Resultados da Estatística Descritiva

\begin{tabular}{|c|c|c|c|c|c|c|c|c|c|}
\hline \multirow{2}{*}{\multicolumn{2}{|c|}{ ASSERTIVAS }} & \multirow{2}{*}{ Média } & \multirow{2}{*}{$\begin{array}{l}\text { Desvio } \\
\text { Padrão }\end{array}$} & \multirow{2}{*}{ Cv } & \multirow{2}{*}{ Sk } & \multirow{2}{*}{$\mathrm{Ku}$} & \multicolumn{3}{|c|}{ Correlação Bivariada } \\
\hline & & & & & & & 1 & 2 & 3 \\
\hline 1. & Eco-Design/Logística Reversa & 4,44 & 1,07 & 0,24 & $-0,60$ & $-1,12$ & 0,69 & & \\
\hline 2. & Desempenho Operacional & 4,63 & 1,13 & 0,24 & $-0,96$ & 0,73 & $0,58^{* *}$ & 0,79 & \\
\hline 3. & Desempenho Ambiental & 4,68 & 1,20 & 0,26 & $-1,21$ & 1,27 & $0,48^{* *}$ & $0,76^{* *}$ & 0,80 \\
\hline \multicolumn{6}{|c|}{ Número medidas } & $\rightarrow$ & 5 & 5 & 5 \\
\hline \multicolumn{6}{|c|}{ Unidimensionalidade ( $\alpha$-Cronbach) } & $\rightarrow$ & 0,73 & 0,84 & 0,86 \\
\hline \multicolumn{6}{|c|}{ Variância Média Extraída (VME) } & $\rightarrow$ & 0,48 & 0,62 & 0,63 \\
\hline \multicolumn{6}{|c|}{ Confiabilidade Composta (CC) } & $\rightarrow$ & 0,82 & 0,89 & 0,90 \\
\hline
\end{tabular}

Nota: $\left.{ }^{* *}\right)$ significante para $(\alpha \leq 0,01)$.

Na diagonal da matriz de correlação, os valores em itálico, representa a raiz quadrada da variância média extraída

Fonte: Dados da pesquisa 


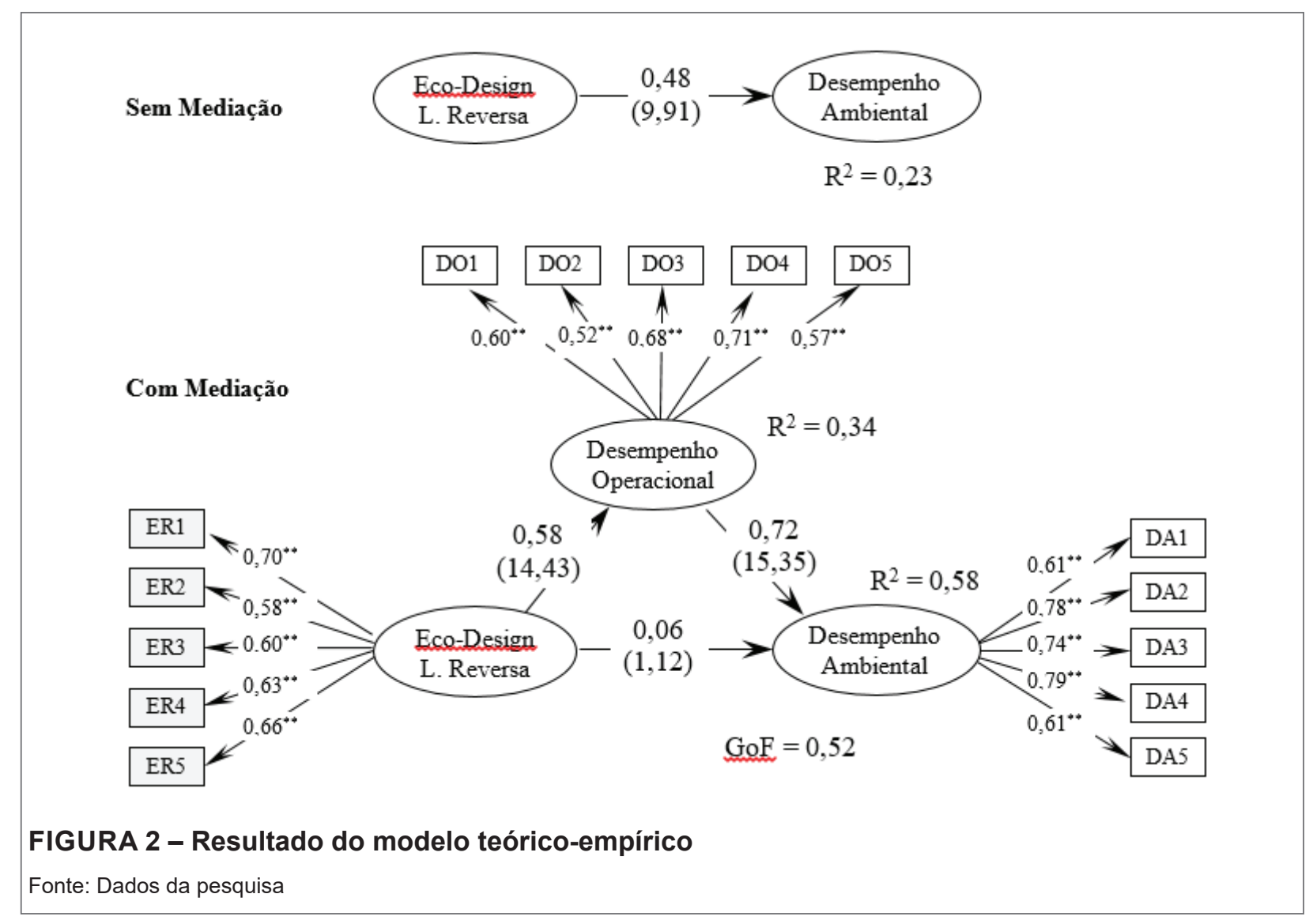

sua vez, influenciou no Desempenho Ambiental com coeficiente estrutural igual a 0,72 e estatística t igual a 15,35 e suportou a hipótese $\mathrm{H}_{3}$.

$\mathrm{Na}$ Tabela 2 são apresentados os coeficientes estruturais, erro padrão e os valores das estatísticas $\mathrm{t}$ das relações do modelo de estimação.

Quanto à adequação do modelo estru- tural, dado pelo coeficiente de determinação $R^{2}$, em relação aos constructos endógenos, obteve-se $\mathrm{R}^{2}$ (médio) igual a 0,46 [0,34 $+0,58) / 2=0,46]$, portanto superior ao recomendado de 0,36 (Cohen, 1988). Para o ajuste global, representado pelo índice GoF, foi obtido o valor igual a 0,52 [GoF = $[()=0,52]$. Portanto, superior ao recomendado de 0,26 (TENENHAUS et al., 2005;

TABELA 2 - Parâmetros do PLS e testes de hipóteses

\begin{tabular}{l|c|c|c|c|c}
\hline \multicolumn{1}{c|}{$\begin{array}{c}\text { RELACIONAMENTO } \\
\text { ESTRUTURAL }\end{array}$} & $\begin{array}{c}\text { COEFICIENTES } \\
\text { ESTRUTURAIS }\end{array}$ & $\begin{array}{c}\text { ERRO } \\
\text { PADRÃO }\end{array}$ & $\begin{array}{c}\text { VALOR } \\
\mathrm{t}\end{array}$ & HIPÓTESE & DECISÃO \\
\hline Modelo - SEM MEDIAÇÃO & & & & & \\
\hline Eco-Design/Logística Reversa $\rightarrow$ D. Ambiental & 0,48 & 0,050 & 9,91 & $\mathrm{H}_{1}$ & Suporta \\
\hline Modelo - COM MEDIAÇÃO & & & & & \\
\hline Eco-Design/Logística Reversa $\rightarrow$ D. Ambiental & 0,06 & 0,057 & 1,12 & $\mathrm{H}_{1 \mathrm{a}}$ & Não suporta \\
\hline Eco-Design/Logística Reversa $\rightarrow$ D. Operacional & 0,58 & 0,040 & 14,43 & $\mathrm{H}_{2}^{* *}$ & Suporta \\
\hline D. Operacional $\rightarrow$ D. Ambiental & 0,72 & 0,048 & 15,35 & $\mathrm{H}_{3}^{*}$ & Suporta \\
\hline
\end{tabular}

$\left({ }^{* *}\right)<0,01$ : level of significance $(t>2,58) ;\left({ }^{*}\right)<0,05$ : level of significance $(t>1,96)$

Fonte: Dados da pesquisa 
WETZELS; ODEKERKEN-SCHRODER; VAN OPEN, 2009).

Prosseguindo na determinação de outros indicadores de qualidade, a validade preditiva $\left(\mathrm{Q}^{2}\right)$, apresentaram valores positivos, mostrando que o modelo refletiu a realidade, isto é, sem erros. Quanto ao tamanho do efeito avaliado pelo $\left(f^{2}\right)$ os constructos apresentaram valores maiores que 0,35 , denotando que o modelo possuia grandes qualidades de predição. $\mathrm{Na}$ Tabela 3 , são mostrados os valores de $\left(\mathrm{Q}^{2}\right)$ e $\left(\mathrm{f}^{2}\right)$.

Portanto, pode-se considerar que houve evidências que os dados coletados tiveram um bom ajuste para o modelo de mensuração desenvolvido.

Para testar a mediação do desempenho operacional entre a Eco-design/Logística reversa e desempenho ambiental, por meio da comparação da magnitude dos coeficientes entre os caminhos (a') e (a) mostrados na Figura I, utilizou-se do teste $z$ de Sobel, que podem ser representados pelos parâmetros: $\mathrm{a}=0,58 ; \mathrm{b}=0,72 ; \mathrm{S}_{\mathrm{a}}=0,040$ e $S_{b}=0,048$ e extraídos da Tabela 2, que aplicados à [Equação I], obteve-se:

teste $\mathrm{z}$ de Sobel $=\quad=10,43 \rightarrow$ valor-p $=0,000$

Portanto, o teste $z$, em nível de significância estatística $(\alpha \leq 0,0 \mathrm{I})$, evidenciou $\circ$ papel mediador do desempenho ambiental na relação entre Eco-design/Logística reversa e desempenho operacional. Quanto a tipologia, segundo abordagem de lacobucci et al. (2007), a mediação foi considerada total.

\section{CONCLUSÕES E SUGESTÕES PARA PROSSEGUIMENTO}

Este estudo foi influenciado pela pouca compreensão que existem nas relações entre eco-design/logística reversa, desempenho operacional e desempenho ambiental. Para tanto, foi estimado um modelo teórico-empírico para um melhor entendimento dessas relações.

Concluiu-se que existiu uma relação, do tipo indireto, em que o desempenho operacional exerceu papel mediador na relação entre eco-design/logística reversa, desempenho operacional. A importância dessa conclusão foi a de evidenciar que as empresas devem envidar esforços nos processos produtivos 'verde' e no eco-design/ logística reversa. Esforços na implantação da produção mais limpa 'ou verde' impactará, no curto prazo, no desempenho operacional. Por sua vez, investimentos em ecodesign/logística reversa, a médio e longo prazo, influenciará em melhor desempenho ambiental. Todavia, outras implicações podem advir dessa conclusão:

a) Implicações para a teoria

A demanda de produtos caminha para uma nova configuração de produção baseada na economia de baixo carbono e conhecimento, de tal modo a valorizar os recursos naturais. Os mercados consumidores podem potencializar incentivos para projetos de produtos que minimizam o impacto ambiental, não somente na sua fabricação, mas durante seu ciclo de vida.

Nesse sentido, pesquisas em produtos

TABELA 3 - Valores dos indicadores da validade preditiva (Q2) e do tamanho do efeito (f2)

\begin{tabular}{l|c|c}
\hline \multicolumn{1}{c|}{ Constructos } & CV RED $\left(\mathrm{Q}^{2}\right)$ & CV COM (f⿱2) \\
\hline Eco-Design/Logística Reversa & 0,23 & 0,23 \\
\hline Desempenho Operacional & 0,19 & 0,43 \\
\hline Desempenho Ambiental & 0,36 & 0,45 \\
\hline Fonte: Dados da pesquisa
\end{tabular}


ecológicos, que causam pouca agressão ao meio ambiente, constantemente estarão alimentando o mercado com tecnologias para a produção mais limpa.A transição da economia baseada no capitalismo industrial para uma economia ambientalmente sustentada é uma realidade. Os recursos naturais, serão considerados pelos seus valores reais e incorporados aos custos dos produtos e serviços que deles se beneficiam.

Por exemplo, a água sempre foi considerada um recurso natural infinito. Todavia, - Brasil, bem como diversos outros países começam a sentir a necessidade de estabelecer limites (SSRH, 2017). Empresas que adotam, sistematicamente, estratégias de projetos para produtos ecológico ou 'verde', em cuja concepção consideram a logística reversa, produção mais limpa e a integração com todas as outras estratégias da organização, terão seus desperdícios reduzidos e ocorrerão melhorias de seus indicadores ambientais. Colocando de outro modo, as empresas, primariamente, terão evoluções crescentes em ecoeficiência, e reduções de materiais e resíduos poluentes como benefícios secundários.

Essas mudanças têm ganhado espaço na competição e na regulação ambiental. Organizações Não Governamentais (ONG's), e agências das Nações Unidas para o meio ambiente, tem abastecidos regularmente a mídia, a sociedade (civil, acadêmica, empresarial) com informações sobre sustentabilidade ambiental, pesquisas, e tecnologias para a produção mais limpa. Os relatórios disponibilizados fornecem uma série de estudos de caso muito úteis mostrando como as empresas desenvolveram projetos ecológicos, implementaram tecnologias para uma produção mais limpa e quais foram os benefícios ambientais e econômicos obtidos.
Salienta-se, todavia, que em muitos casos, as técnicas e tecnologias disponíveis são inadequadas e as soluções devem ser encontradas por meio da investigação científica e do desenvolvimento experimental. Para a eficácia, isso deve estar estreitamente ligado com a avaliação da tecnologia e feeddback apropriados ao longo do processo de pesquisa / design / desenvolvimento. Portanto, a inovação tecnológica apoiada por avaliações simultâneas e políticas públicas eficazes pode ser ferramenta poderosa para alcançar a produção mais limpa, projetada no início do seu ciclo de vida do produto, isto é, no eco-design/logística reversa.

b) Implicações para as práticas gerenciais

Ser sustentável implica em tornar as atividades industriais menos agressivas ao meio ambiente, mais aceitável pela sociedade e, ainda assim, assegurar os lucros e a sobrevivência das organizações. Assim como a filosofia da qualidade total, que tem como meta 'zero defeito' (SCHONBERGER, I 984), a sustentabilidade também almeja a redução total dos resíduos. Tem como meta um alvo móvel, quanto mais se aproxima do objetivo, esse alvo é ampliado para, continuamente, obter avanços para a sustentabilidade ambiental.

A empresa precisa reconhecer o novo meio em que está competindo e mudar de acordo com as necessidades impostas pelo novo cenário. Os avanços na ciências e tecnologias abrirão oportunidades e opções para alcançar a produção limpa. A organização deverá, portanto, absorver a função ecológica, buscando a mudança estrutural da empresa para que seja possível incluir a questão ambiental como fator estratégico. Essa nova estrutura dependerá da pressão do mercado e do compromisso da organização para com o meio ambiente. Salientase, todavia, que deve considerar a atividade 
da empresa, que será determinante para o grau de envolvimento com a questão ambiental; e do seu tamanho, dado que, nas empresas de pequeno porte, o responsável pelo processo produtivo, em geral, também é o responsável pela gestão ambiental.

As questões ambientais devem ser consideradas nas funções existentes nas empresas, evitando o aumento da complexidade organizacional e a criação de mais áreas intermediárias para tomadas de decisão, que acabam por gerar desperdícios de tempo e recursos.

$\mathrm{Na}$ área produtiva, os retrabalhos que requerem a reciclagem ou a eliminação, a espera que pode ocasionar desperdício de energia, superprodução pela maior quantidade de matérias-primas consumidas sem necessidades, movimentação de homens para transporte de estoques em processo e criatividade não utilizada oriundas de sugestões de melhorias dos funcionários, são fatos que ajudam na redução de desperdícios e melhorar o meio ambiente.

Uma variedade de tecnologias de processo, pesquisas acadêmicas, forças do mercado e políticas governamentais podem contribuir, significativamente, para a almejada sustentabilidade ambiental.
Assim, diante dos resultados obtidos e as implicações para a teoria e práticas gerenciais, o desempenho operacional sinalizou ganhos incrementais, de curto prazo, que constituíram o alicerce firme, sem a qual não seria possível vislumbrar metas ambiciosas, de longo prazo, para o desempenho ambiental. Salienta-se, todavia, que investimentos em tecnologias limpas, capacitação em gestão são elementos importantes para que metas de desempenhos operacionais e ambientais sejam logradas pela administração.

Portanto, com o reconhecimento de que os recursos naturais são finitos e a produção deve obedecer aos limites econômicos, tornando assim, viável a ação para alcançar padrões de vida mais sustentável, são sugeridos para prosseguimento desse estudo, investigar nas empresas, especialmente, as de capital aberto, a preferência por tecnologias de produção limpa e seu acompanhamento em termos de desempenho operacional, ou mesmo ambiental. As empresas de capital aberto, em decorrência da instrução 552//4 da Comissão de Valores Mobiliários (CVM), sinalizam a busca da transparência e aprimoramento da gestão de risco, incluindo, a gestão ambiental.

TABELA 1A - Estatística das medidas e escalas

\begin{tabular}{|c|c|c|c|c|c|c|c|c|}
\hline & & CONSTRUCTO / ASSERTIVAS & Média & D.P. & Cv & sk & ku & Carga \\
\hline \multirow{5}{*}{ 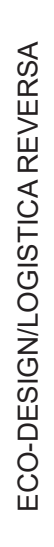 } & ER1 & $\begin{array}{l}\text { A nossa empresa desenvolve o design dos seus produtos } \\
\text { para reduzir o consumo de material e energia }\end{array}$ & 4,14 & 1,74 & 0,42 & $-0,63$ & $-0,86$ & $0,66^{* *}$ \\
\hline & ER2 & $\begin{array}{l}\text { A nossa empresa evita o uso de materiais perigosos ao meio } \\
\text { ambiente nos seus produtos e processos de fabricação }\end{array}$ & 4,76 & 1,42 & 0,30 & $-1,23$ & 0,78 & $0,63^{* *}$ \\
\hline & ER3 & $\begin{array}{l}\text { A nossa empresa atua junto aos seus clientes na implantação } \\
\text { da reciclagem, eliminação de embalagem e na redução de } \\
\text { resíduos }\end{array}$ & 4,44 & 1,46 & 0,33 & $-0,84$ & $-0,09$ & $0,78^{* *}$ \\
\hline & ER4 & $\begin{array}{l}\text { A nossa empresa inclui as habilidades técnicas do pessoal } \\
\text { nos aspectos ambientais }\end{array}$ & 4,80 & 1,28 & 0,27 & $-0,99$ & 0,24 & $0,77^{* *}$ \\
\hline & ER5 & $\begin{array}{l}\text { A nossa empresa prevê a logística reversa no design dos } \\
\text { produtos como desmontagem, reuso, reciclagem e descarte } \\
\text { correto }\end{array}$ & 4,05 & 1,75 & 0,43 & $-0,55$ & $-0,97$ & $0,59^{* *}$ \\
\hline
\end{tabular}




\begin{tabular}{|c|c|c|c|c|c|c|c|c|}
\hline \multirow{5}{*}{ 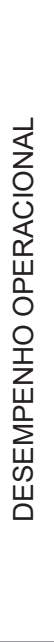 } & DO1 & $\begin{array}{l}\text { A nossa empresa diminuiu a taxa de sucatas ao participar da } \\
\text { GSCM nos últimos } 2 \text { anos }\end{array}$ & 4,38 & 1,57 & 0,36 & $-1,20$ & 0,68 & $0,76^{* *}$ \\
\hline & DO2 & $\begin{array}{l}\text { Na nossa empresa a qualidade dos produtos e serviços co- } \\
\text { mercializados melhorou com a gestão ambiental nos últimos } \\
2 \text { anos }\end{array}$ & 4,60 & 1,44 & 0,31 & $-0,94$ & $-0,28$ & $0,87^{* *}$ \\
\hline & DO3 & $\begin{array}{l}\text { A nossa empresa melhorou a utilização da capacidade ope- } \\
\text { racional (melhor uso dos recursos produtivos) nos últimos } 2 \\
\text { anos }\end{array}$ & 4,65 & 1,46 & 0,31 & $-0,97$ & 0,02 & $0,84^{* *}$ \\
\hline & DO4 & $\begin{array}{l}\text { Na nossa empresa as metas de minimizar e eliminar as não } \\
\text { conformidades dos produtos foram atingidas nos últimos } 2 \\
\text { anos }\end{array}$ & 4,58 & 1,45 & 0,32 & $-1,42$ & 0,85 & $0,65^{* *}$ \\
\hline & DO5 & $\begin{array}{l}\text { A nossa empresa melhorou a qualidade dos registros, aná- } \\
\text { lises e soluções das reclamações dos clientes nos } 2 \text { últimos } \\
\text { anos }\end{array}$ & 4,93 & 1,31 & 0,27 & $-1,36$ & 1,76 & $0,78^{* *}$ \\
\hline \multirow{5}{*}{ 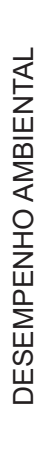 } & DA1 & $\begin{array}{l}\text { A nossa empresa reduziu as emissões de poluentes ao ado- } \\
\text { tar a gestão ambiental nos últimos } 2 \text { anos }\end{array}$ & 4,71 & 1,48 & 0,31 & $-0,83$ & $-0,31$ & $0,79^{* *}$ \\
\hline & DA2 & $\begin{array}{l}\text { A nossa empresa conseguiu reduzir o uso de águas residuais } \\
\text { com a implantação da gestão ambiental nos últimos } 2 \text { anos }\end{array}$ & 4,49 & 1,66 & 0,37 & $-1,07$ & 0,45 & $0,79^{* *}$ \\
\hline & DA3 & $\begin{array}{l}\text { A nossa empresa reduziu os resíduos sólidos ao efetuar a } \\
\text { gestão ambiental nos últimos } 2 \text { anos }\end{array}$ & 4,53 & 1,55 & 0,34 & $-1,14$ & 0,56 & $0,78^{* *}$ \\
\hline & DA4 & $\begin{array}{l}\text { A nossa empresa diminuiu a frequência de acidentes ambien- } \\
\text { tais com a gestão ambiental nos últimos } 2 \text { anos }\end{array}$ & 4,83 & 1,60 & 0,33 & $-1,10$ & 0,49 & $0,78^{* *}$ \\
\hline & DA5 & $\begin{array}{l}\text { A nossa empresa com a adoção da gestão ambiental melho- } \\
\text { rou as atividades operacionais nos últimos } 2 \text { anos }\end{array}$ & 4,84 & 1,26 & 0,26 & $-1,53$ & 2,03 & $0,84 *$ \\
\hline
\end{tabular}

Nota: $\left(^{\star *}\right)$ significante para $(\alpha \leq 0,01)$

Fonte: Dados da pesquisa 
BARON, R. M.; KENNY, D. A. The moderator-mediator variable distinction in social psychological research: Conceptual, strategic, and statistical considerations. USA: Journal of Personality and Social Psychology, v. 5I, pp. II73I | 82, 1986.

BOWERSOX, D. J.; CLOSS, D. J.; COOPER, M. B.; BOWERSOX, J. C. Gestão logística da cadeia de suprimentos. Porto Alegre: Editora Bookman. 4 ${ }^{\mathrm{a}}$ Edição, 2014, 455p.

BOWERSOX, D. J.; CLOSS, D. J.; COOPER, M. B. Gestão logística de cadeia de suprimentos. Porto Alegre: Editora Bookman. 2006, 529p.

CHASE, R. C.;JACOBS, F. R.;AQUILANO, N. J.Administração da produção para a vantagem competitiva. Porto Alegre: Editora Bookman, $10^{a}$ Edição, 2006, 724p.

COHEN, J. Statistical Power Analysis for the Behavioral Sciences (2nd ed.). Hillsdale, NJ: Erlbaum, 1988.

CORRÊA, H. L.; CORRÊA, C. A. Administração de produção e operações. Manufatura e serviços: uma abordagem estratégica. São Paulo: Editora Atlas, $3^{\mathrm{a}}$ Edição, 2012, 680p.

DEMING, W. E. Out of the Crisis. The MIT Press, Cambridge, MA, 2000.

FORNELL, C.; LARCKER, D. F. Evaluating structural equation models with unobservable variables and measurement error. USA: Journal of Marketing Research, 39-50. 1981.

HALL, R. H. Organizações: estruturas, processos e resultados. São Paulo: Pearson, 8a. Edição, 2004, 322p.

HENSELER, J.; SARSTED, M. Good-
ness-of-Fit Indices for Partial Least Squares Path Modeling. USA: Computational Statistics, 28, pp. 565-580, 2012.

IACOBUCCI, D.; SALDANHA, N.; DENG, X. A meditation on mediation: evidence that structural equations models perform better than regressions. USA: Journal of Consumer Psychology, v. I7, n. 2, pp. I40-I54. 2007.

ISOI4006. Environmental management systems - guidelines for incorporating ecodesign. 201I. Acessado em 2I de julho de 2017 de: https://www.iso.org/ standard/4324I.html.

KIURSKI, J. S.; MARIC, B. B.; OROS, I. B.; KECIC, V. S. The ecodesign practice in Serbian printing industry. USA: Journal of Cleaner Production. V. 149, PP. I200I209, 2017.

KRUWET, A.; ZUSSMAN, E.; SELIGER, G. Systematic integration of design for recycling into product design. UK: International Journal of Production Economics, Oxford, v. 38, p. 15-22, 1995.

LARDEREL, J.A. Cleaner Production: Introduction. In UNEP Industry and Environmental. USA: Cleaner Production. P. 5, januaryjune $200 \mathrm{I}$.

LEITE, P. R. Logística Reversa: meio ambiente e competitividade. $3^{\mathrm{a}}$ Edição. São Paulo: Saraiva, 2017, 360p.

LINTON,J. D.; KLASSEN, R.;JAYARAMAN, V. Sustainable supply chains: An introduction. USA: Journal of Operations Management, 25(6), 2007, pp. 1075-1082.

MOLLENKOPF, D. A.; CLOSS, D. J. The hidden value in reverse logistics. USA: Supply chain management review. v. 9, pp. 34-43, 2005.
NEELY,A. Measuring business performance. London: The Economist in Association with Profile Books, 1998.

NINLAWAN, C.; SEKSAN, P.;TOSSAPOL, K.; PILADA, W. The implementation of green supply chain management practices in electronics industry. Hong Kong: International Multiconference of Engineers and Computer Scientists, 2010.

PNRS - Política Nacional de Resíduos Sólidos. Brasília: Centro de documentação e informação, $2^{\mathrm{a}}$ Edição, 2012.

REN, Y. The strategic implications of Japanese environmental management. USA: International review for environmental strategies. v. 2, n. I. 200 I, Pp. I27-I47. ROMM, J. Lean and clean management: how to boost profit and productivity by reducing pollution. New York: Kodansha International, 1994.

SANT'ANNA, F. S. P.; CAMPOS, L. M. S.; PEREIRA, G. R. Produção mais limpa $(\mathbf{P}+\mathbf{L})$. In Gestão empresarial e sustentabilidade. Coordenador:Arlindo Philippi Jr. São Paulo: Editora Manole, Pp. 675-701, 2017.

SCHONBERGER, R. J. Técnicas Industriais Japonesas. São Paulo, Pioneira, 1984.

SSRH - Secretaria de Saneamento e Recursos Hídricos do Estado de São Paulo. Acessado em 24/julho/2017 de http:// 43.1 07.108.83/sigrh/cobranca/perguntas.html

SRIVASTAVA, S. K. Green supplychain management: a state-ofthe-art literature review. USA: International journal of management reviews, v. 9, n. I, 2007. pp. 53-80. 
TENENHAUS, M.; VINZI, V. E.; CHATELIN, Y.; LAURO, C. PLS-Path Modeling. USA: Computational Statistics \& Data Analysis, v. 48, n. I, pp. I59-205, 2005.

TIBBEN-LEMBKE, R. S. Life after death: reverse logistics and the product life cycle. USA: International jornal of physical distribution \& logistics management. V. 32, n. 3, pp. 223-244, 2002.

TOEPFER, K. The global framework for changing current production and consumption patterns has been set - the time for action is now. In UNEP Industry and Environmental. USA: Cleaner Production. editorials, p. 3, januaryjune 200 I.

UNEP - United Nations Environment Programme. Cleaner Production sixth international high-level seminar. Montreal: Industry and En- vironment. January-june, 200I. VIJAYVARGY, Lokesh; AGARWAL, Gopal. Empirical investigation of green supply chain management practices and their impacto $n$ organizational performance. Indian: The IUP jornal of supply chain management. v. XI, n. 4, Pp. 25-42, 2014.

WETZELS, M.; ODEKERKEN-SCHRODER, G.; VAN OPPEN, C. Using PLS Path Modeling For Assessing Hierarchical Construct Models: Guidelines and Empirical Illustration. USA: MIS Quarterly, v. 33, n. I, Pp. I77-195, 2009.

ZHU Q.; SARKIS, J. Relationships between operational practices and performance among early adopters of green supply chain management practices in Chinese manufacturing enterprises. USA: Journal of Operations Management, v. 22, n. 3, pp. 265-289, 2004.
The modera-

ting effects of institutional pressures on emergent green supply chain practices and performance. USA: International journal of production research. v. 45, ns. I8-19, pp. 4333-4355, 2007.

ZHU, Q.; GENG,Y.; FUJITA,T.; HASHIMOTO, S. Green supply chain management in leading manufacturers: Case studies in Japanese large companies. USA: Management Research News, v. 33, n. 4, pp. 380-392, 2010.

APÊNDICE I: Medidas e Escalas

Todas as assertivas foram medidas pela escala de Discordância / Concordância com seis graus de importância que variava de Discordo Totalmente $(D T=I)$ a Concordo Totalmente $(\mathrm{CT}=6)$.A média aritmética, desvio padrão, assimetria (sk), alongamento (ku) e carga fatorial. 